\title{
Coulomb Blockade and Disorder in 2D Quantum Dot Arrays
}

\author{
Heinz-Olaf MüLleR*1 ${ }^{* 1}$ David A. Williams and Hiroshi MizUTA*2 \\ Hitachi Cambridge Laboratory, Cavendish Laboratory, Madingley Road, Cambridge, CB3 OHE, U. K.
}

(Received April 27, 2000; accepted for publication June 9, 2000)

We investigate the influence of both size disorder and background charge disorder, on the Coulomb blockade voltage of two-dimensional arrays of metallic quantum dots for varying array size. Both the mean blockade voltage and its variation are considered for several array sizes with varying degrees of disorder. Design rules for devices using those arrays are derived.

KEYWORDS: device physics, Coulomb blockade, quantum dot array, size disorder, background charge

Today's technology provides the means for the study of Coulomb interactions between single electrons and/or quantum mechanical tunneling. Furthermore, the difference between the characteristic length scales of these two effects by approximately a factor of ten (depending on the specific setup) allows the construction of devices exploiting strong Coulomb interaction and weak tunneling. In this regime the electron number becomes a good quantum number and is directly connected to the dominant energy scale. This paves the way to a quasi-classical description in terms of the number of extra charges, known as the "orthodox theory of Coulomb blockade". 1) The parameters of this description are resistances and capacitances, which reflect tunneling probabilities and the Coulomb interaction, respectively. The rate equation at the core of the "orthodox theory" proved very helpful for the understanding of devices such as the single-electron transistor, ${ }^{2)}$ the multiple-tunnel junction, ${ }^{3)}$ or the Coulombblockade thermometer. ${ }^{4)}$ All these systems exploit the effect of Coulomb blockade, which leads to a total suppression of the current in suitable systems for sufficiently low bias voltage. ${ }^{5)}$

In recent years, interest in the Coulomb blockade phenomenon has turned from exploration to application, and a number of devices have been proposed. These include sensors, such as the electrometer ${ }^{6)}$ and the thermometer, ${ }^{4)}$ a current standard, ${ }^{7)}$ and digital memory ${ }^{8)}$ and logic. ${ }^{9)}$ Following this development, questions concerning fabrication yield, reliability, and fault tolerance become more and more important.

In this paper we use simulation tools ${ }^{10)}$ based upon the "orthodox theory" to assess the effect of disorder on the device performance of Coulomb blockade systems. We study the Coulomb blockade voltage $V_{\mathrm{b}}$, i.e. the threshold voltage required to overcome current suppression, of two-dimensional arrays of quantum dots. This definition of $V_{\mathrm{b}}$ is based on zero-temperature operation, as this is the only temperature at which current suppression is complete, however the parameter itself describes the essential features of the device characteristics for a range of temperatures, $k_{\mathrm{B}} T \ll e V_{\mathrm{b}}$. The array size varies from $1 \times 1$ to $5 \times 5$. Hence, single dot and onedimensional arrays are studied as special cases. The inset of Fig. 1 gives an schematic impression of a $3 \times 2$ array.

We simulate size disorder by varying the dot's lateral aspect ratio, and background charge disorder with variable voltage sources charging the self capacitances of the dots (see inset of Fig. 1). The disorder values in this paper are given as percent.

\footnotetext{
${ }^{* 1}$ E-mail address: hom@phy.cam.ac.uk

*2E-mail address: mizuta@ phy.cam.ac.uk
}

For size disorder, both dimensions of the rectangular dots are varied independently. The regular dot size is $5 \times 5 \mathrm{~nm}^{2}$ and the dots' centers lie on a $7 \mathrm{~nm}$ square grid so that $100 \%$ disorder would correspond to $2 \mathrm{~nm}$ variation in each direction. ${ }^{10)}$ Background charge disorder allows for a restricted variation of the influenced charge from dot to dot, maximal $e$. These charge values are mapped into the range $[-e / 2: e / 2]$ to allow for relaxation via tunneling.

In our study we step through the whole range of either size or background charge disorder for all accessible array sizes from $1 \times 1$ to $5 \times 5$. Ten samples with equal parameters are used for statistical evaluation. The limitation of this number is primarily due to computing time constraints. From the calculated data we extract the average value of $V_{\mathrm{b}},\left\langle V_{\mathrm{b}}\right\rangle$ and its variation $\left\langle\left\langle V_{\mathrm{b}}\right\rangle\right\rangle$. These two parameters alone do not allow for the full characterization of a Coulomb blockade device, but are an essential part of it. Appropriate design rules can be derived for specific device applications.

The main limitations of our approach were presented already in ref. 10. They result from the use of the quasiclassical "orthodox model" and the determination of its parameters, capacitances and resistances. As pointed out ear-

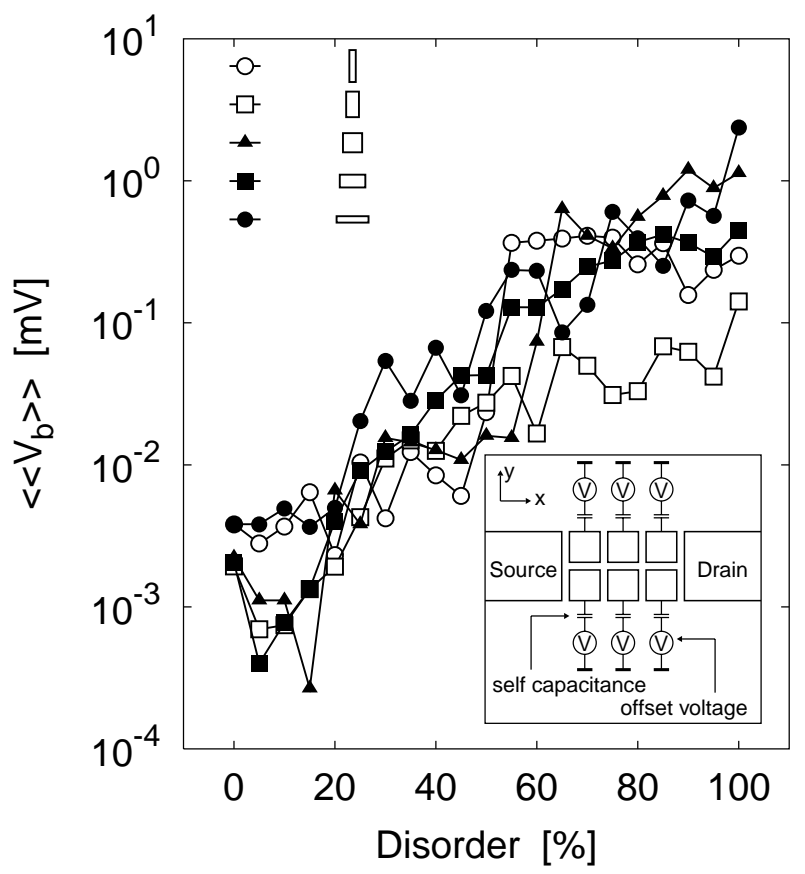

Fig. 1. Dependence of the blockade voltage variation on the degree of size disorder for dot arrays of different length/width ratio. Inset: the case of the $3 \times 2$ array of quantum dots as studied in this work. The current transport follows the $x$-direction. A voltage source is attached to every dot self-capacitance in order to simulate the effect of background charges. 
lier, ${ }^{11)}$ the resistance values do not influence the Coulomb blockade voltage $V_{\mathrm{b}}$. The determination of the latter is a purely electrostatic problem.

Coulomb blockade in complex systems has attracted some interest during the last few years. Some work, of both experimental and theoretical nature, was sparked off by ref. 12, which studied the behavior of the tunneling current for bias slightly above $V_{\mathrm{b}}$ and the influence of background charges. The value of the Coulomb blockade voltage $V_{\mathrm{b}}$ was investigated in one-dimensional arrays, especially under the influence of background charge disorder. ${ }^{11,13,14)}$ Size fluctuations of stacked quantum dot arrays were investigated using photoluminescence spectroscopy. ${ }^{15)}$

In the main part of Fig. 1 we display the results for the blockade voltage variation, $\left\langle\left\langle V_{\mathrm{b}}\right\rangle\right\rangle$. The plot shows this variation as function of the degree of size disorder for several array geometries. An exponential increase of the variation with increasing disorder is observed. It turns out that $\left\langle\left\langle V_{\mathrm{b}}\right\rangle\right\rangle$ depends much more strongly on the degree of disorder than the actual array layout, the latter dependence not being significant in our data. Therefore it must be doubted that the array layout is a helpful design parameter when it comes to reduction of the blockade voltage variation. Figure 1 also shows the level of blockade voltage variations which is small compared to $\left\langle V_{\mathrm{b}}\right\rangle$ (compare Figs. 2 and 3). This justifies the analysis below. Note that we found a similar magnitude of blockade voltage variations as function of background charge disorder, but lacking the clear disorder dependence.

In Fig. 2 we display $\left\langle V_{\mathrm{b}}\right\rangle$ for arrays of different size and shape as well as different degrees of disorder. Figure 2(a) shows that the length/width ratio of the array hardly influences the blockade voltage in the case of size disorder. There
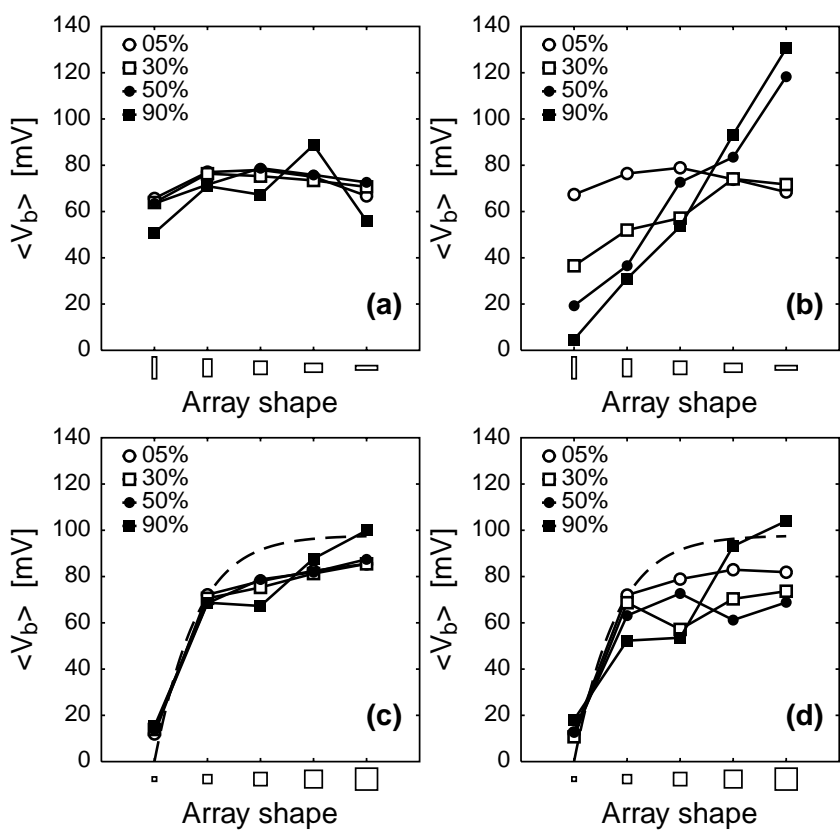

Fig. 2. Mean value of the Coulomb blockade voltage $V_{\mathrm{b}}$ for different array size and disorder: (a) size disorder in arrays of size $1 \times 5,2 \times 4,3 \times 3,4 \times 2$, and $5 \times 1$ (from left to right); (b) background charge disorder in similar arrays; (c) size disorder in $1 \times 1,2 \times 2,3 \times 3,4 \times 4$, and $5 \times 5$ arrays; (d) background charge disorder in similar arrays. Different curves correspond to different levels of disorder. The dashed line in (c) and (d) corresponds to the analytical result of Melsen et al. for the regular, one-dimensional array. is some fluctuation for high disorder, but otherwise the curves are almost flat and atop of each other. The situation is different for background charge disorder, Fig. 2(b), where a flat curve is observed for very small disorder only. If the disorder level increases, $\left\langle V_{\mathrm{b}}\right\rangle$ drops for the short and wide array, but increases considerably for the narrow long one. The latter can be explained in terms of the charge pinning. ${ }^{11)}$ The former is not too hard to understand either: $V_{\mathrm{b}}$ will correspond to the lowest blockade voltage of a number of current paths which in turn increases with the width of the array. Approximately square-like arrays appear to be preferable from the design point of view since $\left\langle V_{\mathrm{b}}\right\rangle$ remains unaffected by the degree of disorder.

Therefore we display the results for square-like arrays in Figs. 2(c) and 2(d). Again, Fig. 2(c) shows the influence of size disorder on $\left\langle V_{\mathrm{b}}\right\rangle$, whereas Fig. 2(d) is devoted to background charge disorder. Besides the relative independence on the degree of disorder (similar to Fig. 2(a)) the dominant feature in Fig. 2(c) is the sharp increase of $\left\langle V_{b}\right\rangle$ for small arrays. Similar behavior was found for truly one-dimensional arrays without disorder. ${ }^{11)}$ Using the capacitance values of the regular case $\left(C=0.49 \mathrm{aF}, C_{\mathrm{g}}=1.26 \mathrm{aF}\right.$, critical array length $N_{\mathrm{c}}=2.5 \sqrt{C / C_{\mathrm{g}}} \approx 1.6$ ) we plot their result (4.4) as dashed line in Figs. 2(c) (and 2(d)). The good overall correspondence suggests two conclusions: (i) the transport is dominated much more by the length of the array than the degree of disorder, and (ii) the transport is rather one-dimensional. Figure 2(d) displays a similar comparison for the case of background charge disorder. Again, the initial increase in $\left\langle V_{\mathrm{b}}\right\rangle$ for all different degrees of disorder is well predicted by the dashed curve. The saturation value of $\left\langle V_{\mathrm{b}}\right\rangle$ reached for large arrays depends more strongly on the degree of disorder than that of Fig. 2(c), but our data does not allow for detailed analysis. As opposed to Fig. 2(c) transport is probably not along simple one-dimensional channels. In general, we find a strong size/shape dependence of $\left\langle V_{\mathrm{b}}\right\rangle$, which is in stark contrast to its variation (see Fig. 1).

In Fig. 3 we explicitly consider the array width dependence of $\left\langle V_{\mathrm{b}}\right\rangle$ and $\left\langle\left\langle V_{\mathrm{b}}\right\rangle\right\rangle$. While there is only a weak influence in the case of size disorder (a), harmonization is observed for background charge disorder in wider arrays: $\left\langle V_{\mathrm{b}}\right\rangle$ becomes independent of the level of disorder. As discussed above, this averaging is attributed to the increasing number of current paths, while at the same time Coulomb blockade breakdown is prohibited by the array length. For arrays of three dots and wider the influence of disorder on the value of $V_{\mathrm{b}}$ is only weakly dependent on the actual degree of disorder. Figures 3(c) and 3(d) confirm the outcome of Fig. 1 insofar as the variation $\left\langle\left\langle V_{\mathrm{b}}\right\rangle\right\rangle$ depends much more strongly on the degree of disorder than the array layout.

In conclusion we investigated both the Coulomb blockade voltage and its variation for quantum dot arrays of different size under the influence of disorder. We studied both size disorder and background charge disorder, but both forms independently of each other. We found that square-like arrays of sizes larger than the "critical size" $N_{\mathrm{c}}$ of ref. 11 are favorable from the design point of view. They display profound stability against both background charge disorder and size disorder. In addition averaging causes harmonization of the blockade voltage despite varying levels of background charge disorder in these arrays. We suppose also that transport in size disordered 


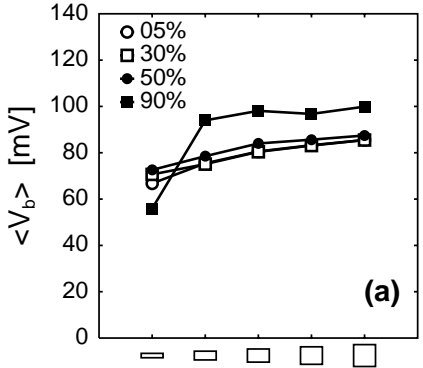

Array shape

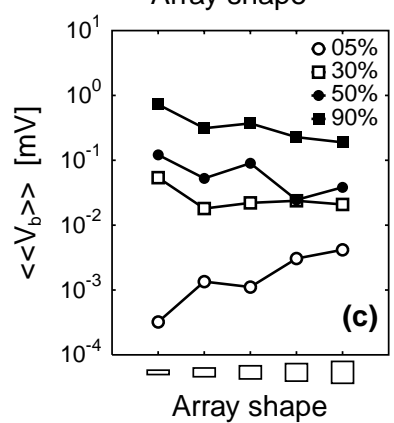

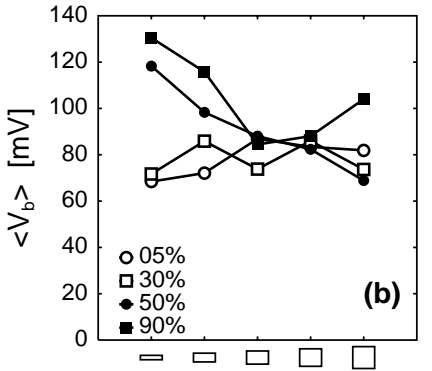

Array shape

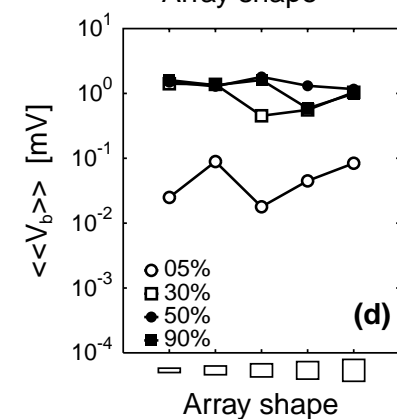

Fig. 3. $\left\langle V_{\mathrm{b}}\right\rangle$ and $\left\langle\left\langle V_{\mathrm{b}}\right\rangle\right\rangle$ for five dots long arrays of varying width from 1 to 5 dots (left to right): (a) $\left\langle V_{\mathrm{b}}\right\rangle$ for different values of size disorder; (b) similar for background charge disorder; (c) $\left\langle\left\langle V_{\mathrm{b}}\right\rangle\right\rangle$ for size disorder; and (d) background charge disorder.

arrays remains predominantly one-dimensional, in contrast to arrays infested with background charges. The variation of the blockade voltage generally depends much more strongly on the degree of disorder than the geometry of the array.
This work was performed within the ESPRIT MEL-ARI project FASEM (Fabrication and Architecture of SingleElectron Memories).

1) D. V. Averin and K. K. Likharev: Mesoscopic Phenomena in Solids, in Modern Problems in Condensed Matter Sciences, eds. B. L. Altshuler, P. A. Lee and R. A. Webb (Elsevier, Amsterdam, 1991) Vol. 30, pp. 173271.

2) T. A. Fulton and G. J. Dolan: Phys. Rev. Lett. 59 (1987) 109.

3) K. Nakazato and H. Ahmed: Adv. Mater. 5 (1993) 668.

4) J. P. Pekola, K. P. Hirvi, J. P. Kauppinen and M. A. Paalanen: Phys. Rev. Lett. 73 (1994) 2903.

5) Single Charge Tunneling: Coulomb Blockade Phenomena in Nanostructures, eds. H. Grabert and M. H. Devoret (Plenum, New York and London, 1992) NATO ASI Series B: Physics, Vol. 294.

6) A. N. Korotkov, D. V. Averin, K. K. Likharev and S. A. Vasenko: Proc. 4th Int. Conf. SQUID'91, eds. H. Koch and H. Lübbig (Springer, Berlin, Heidelberg, 1991) pp. 45-59.

7) J. M. Martinis, M. Nahum and H. D. Jensen: Phys. Rev. Lett. 72 (1994) 904.

8) K. Nakazato, R. J. Blaikie and H. Ahmed: J. Appl. Phys. 75 (1994) 5123.

9) K. Tsukagoshi, B. W. Alphenaar and K. Nakazato: Appl. Phys. Lett. 73 (1998) 2515.

10) H.-O. Müller, K. Katayama and H. Mizuta: J. Appl. Phys. 84 (1998) 5603.

11) J. A. Melsen, U. Hanke, H.-O. Müller and K. A. Chao: Phys. Rev. B 55 (1997) 10638.

12) A. A. Middleton and N. S. Wingreen: Phys. Rev. Lett. 71 (1993) 3198.

13) P. Delsing: Single Charge Tunneling: Coulomb Blockade Phenomena in Nanostructures, eds. H. Grabert and M. H. Devoret (Plenum, New York and London, 1992) NATO ASI Series B: Physics, Vol. 294, pp. 249-74.

14) P. Lafarge, J. J. Meinndersma and J. E. Mooij: Czech. J. Phys. 46 (1996) 2361.

15) A. Endoh et al.: Jpn. J. Appl. Phys. 38 (1999) 1085. 